\title{
COMPETENCIAS GERENCIALES PARA INGENIEROS
}

MANAGEMENT SKILLS FOR ENGINEERS

HABILIDADES DE GERENCIAMENTO PARA ENGENHEIROS

\author{
Jennifer Catalina Murcia Rodríguez \\ Jennifer.murcia@uniminuto.edu \\ Corporación Universitaria Minuto de Dios- UNIMINUTO \\ Bogotá D.C - Colombia
}

Diagramación e llustración de Portada

Sindy Catherine Charcas Ibarra

Encuentre este artículo en:

http://revistas.uniminuto.edu/index.php/IYD

Para citar este artículo / To cite this article

Murcia- Rodríguez, J. (2020). Competencias gerenciales para ingenieros. Inclu-

sión \& Desarrollo, 7 (2), pp. 101-110
Fecha de recepción: 20 de febrero de 2020

Fecha de aceptación: 20 de mayo de 2020

Fecha de publicación: 30 de junio de 2020 


\section{RESUMEN}

Objetivo. Analizar la importancia de las competencias gerencialesen los profesionales de las áreas de ingenierías, estableciendo las más representativas para el desarrollo de proyectos empresariales y sociales.

Metodología. La investigación se desarrolló bajo el enfoque cualitativo de tipo descriptivo con un componente descriptivo, para realizar indagación documental sobre características de lascompetencias gerenciales y su pertinencia en el campo ingenieril, con el fin de fortalecerlas para el fomento y fortalecimiento de proyectos productivos, organizacionales y sociales, en beneficio del crecimiento económico de las empresas y el mejoramiento de la calidad de vida de las comunidades que requieren de propuestas investigativas, para el cubrimiento de sus necesidades cotidianas.

Conclusión. A partir de la revisión y análisis documental, se encontraron más de treinta competencias gerenciales, así como fases para el desarrollo de proyectos; de esta manera se pudo determinar qué competencias son las pertinentes, para abordar cada fase y así dar logro a la formulación, ejecución y seguimiento a los mismos, el rol que tiene el ingeniero para analizar las necesidades del entorno empresarial y social y así diseñar y gestionar los proyectos.

Palabras clave: ingeniería, competencias, innovación, competitividad, desarrollo empresarial, gerencia.

\section{ABSTRACT}

Objective. Analyzetheimportanceofmanagementskills in professionals in theengineeringareas, establishingthemostrepresentativeonesforthedevelopmentofbusiness and social projects.

Methodology. The research was developed under the qualitative approach of a descriptive type with a descriptive component, to carry out documentary research on the characteristics of managementcompetences and their relevance in the engineering field, in order to strengthen these competencies, for the promotion and strengthening of productive projects, organizational and social, in benefit of the economic growth of the companies and the improvement the quality of life of communitiesthatrequire research proposals to cover their daily needs.

Conclusion. From the documentary review and analysis, more than thirty management competencies were found, as well as phases for the development of projects, in this way it was possible to determine which competencies are relevant, to address each phase and thus achieve the formulation, execution and monitoring of these, and the role of the engineer to analyze the needs of the business and social environment and in this waydesign and manage projects.

Keywords: engineering, skills, innovation, competitiveness, business development, management.

\section{SUMÁRIO}

Objetivo. Analisar a importância das competências gerenciais em profissionais das áreas de engenharia, estabelecendo as mais representativas para o desenvolvimento de negócios e projetos sociais.

Metodologia. A pesquisa foi desenvolvida sob a abordagem qualitativa do tipo descritivo, com um 
componente descritivo, para realizar pesquisa documental sobre as características das competências gerenciais e sua relevância no campo da engenharia, a fim de fortalecê-las para a promoção e fortalecimento de projetos organizacionais produtivos. e social, em benefício do crescimento econômico das empresas e da melhoria da qualidade de vida das comunidades que necessitam de propostas de pesquisa, para atender às suas necessidades diárias.

Conclusão. A partir da revisão e análise documental, foram encontradas mais de trinta competências gerenciais, além de fases para o desenvolvimento dos projetos; Dessa forma, foi possível determinar quais competências são pertinentes, abordar cada fase e, assim, alcançar a formulação, execução e monitoramento das mesmas, o papel que o engenheiro tem para analisar as necessidades do ambiente comercial e social e, assim, projetar e gerenciar projetos.

Palavras chave: engenharia, habilidades, inovação, competitividade, desenvolvimento de negócios, gestão. 


\section{Introducción}

El mundo empresarial se enfrenta hoy en día a nuevos retos de competitividad, gracias a las nuevas tendencias tecnológicas que están inmersas en todos los procesos organizacionales con el fin de agilizar funciones y generar atenciones al cliente más rápidas y efectivas, esto les exige a los profesionales investigar y mejorar continuamente sus conocimientos, que les permita aportar a la innovación y desarrollo en las empresas. Actualmente la dinámica laboral ha cambiado, antes era importante la fuerza física en empleados, ahora es necesario fortalecer el capital humano en tecnologías blandas, para que contribuya al logro de los objetivos, analizando y estudiado de manera asertiva los esquemas de los mercados y las necesidades de los usuarios y consumidores, así como las nuevas formas de producción y transformación de materia prima, entre otros factores.

La concreción de estrategias de desarrollo sostenible demanda ingenieros competentes en la formulación, ejecución y evaluación de proyectos para las diversas áreas de la sociedad. El fortalecimiento de las competencias gerenciales para proyectos(Roldán, 2012), exige la identificación de necesidades y oportunidades desde una mirada multidisciplinar. En este mismo sentido la formación de especialistas en proyectos requiere de un modelo de enseñanza que privilegie la gestión social, participación y desarrollo comunitario.

Es por esto, que los profesionales y en especial los ingenieros deben estar en la capacidad de formular y ejecutar proyectos de inversión, productivos o de investigación para el desarrollo de nuevos productos y servicios, generando a su vez innovación, como un elemento importante en el campo de la competitividad(Caballero, 2001); por lo tanto es importante que los ingenieros manejen y conozcan las competencias gerenciales para que les permitan abordar un tema importante que servirá como una idea empresarial, donde necesitará el análisis para la selección de recursos, liderazgo de equipos de trabajo y evaluación de una oferta y demanda; las instituciones de educación superior forman ingenieros desde lo disciplinar, transfiriéndoles conocimiento con teorías administrativas sobre procesos y procedimientos, aspectos de calidad y sistemas de gestión, pero toda esa información es recordada de manera parcial, así sucede con las competencias gerenciales, porque son un gran número que aprendérselas de memoria, resulta muy difícil.

Existen especializaciones en gerencia de proyectos, en donde los estudiantes conocen más de treinta competencias, que son las que forman a un especialista en proyectos, en este programa académico, se manejan módulos de aprendizaje en donde continuamente se trabajan las competencias, pero ¿si se recordarán todas?, de acuerdo con las experiencias de los docentes, se evidencia que sólo un $5 \%$ de los ingenieros una vez graduados recuerdan algunas de ellas. Lo más importante no es memorizarlas, lo realmente relevante es que los egresados las recuerden y las pongan en práctica de acuerdo con los contextos que requieren de habilidades gerenciales, que pueden ir desde identificar una oportunidad de inversión, hasta evaluar su viabilidad, esas mismas habilidades majeándolas asertivamente, permitirá una toma de decisión rentable para la nueva propuesta empresarial.

Entonces, desde el aula es muy importante que el ingeniero, reconozca cada una de las competencias(The In stitute for Business and Finance Research, 2012), y que, con el acompañamiento del docente, identifique cuáles son las que orientan hacia la formulación, la planificación, seguimiento y ejecución de un proyecto; la base para este aprendizaje se centra en poner en práctica todos los conocimientos, para que, desde ejercicios prácticos, permanezca a mediano y largo plazo la información sobre competencias y su importancia en el desarrollo laboral. 
Es importante resaltar, que en el campo de las capacitaciones, se manejan guías como New Perspectiveson Evaluation and Certifying the Competencesof Higher Education Graduates, Project management standards, project Management In Development Cooperation, NonGovernmental Organizations, entre otros, donde exponen competencias para trabajar los modelos de formulación y evaluación de proyectos, es decir existen organizaciones que orientan sobre el tipo de habilidades que deben manejar los ingenieros, pero son tantas que un egresado especialista en proyectos, no las puede recordar ni mucho menos asociarlas ante diferentes situaciones que se enfrente.

Por otra parte, en el contexto internacional existe el Proyecto TuringLatin- América, basado en los fundamentos filosóficos de Bédard, en donde identificó 47 competencias gerenciales (Castrillón, Cabeza, \& Lombana, 2015)que son percibidas de manera diferente por académicos y empleadores; esta experiencia permite identificar las más importantes para el contexto colombiano y a partir de ellas buscar estrategias que fortalezcan la formación de los profesionales de las diversas áreas de la administración.

De acuerdo con lo anterior,es necesario que el docente caracterice las competencias(Contraloría General de la Respública, 2010), seleccione las más representativas teniendo en cuenta la dinámica del mercado y sus exigencias, evalúe al grupo de estudio y determine las metodologías de enseñanza apropiadas para fortalecerlas, de esta manera lograr que los ingenieros, una vez culminen sus estudios, se sientan en la capacidad de formular proyectos gerenciales, liderar un grupo y tener la capacidad de tomar decisiones asertivas, importantes para el crecimiento de una empresa y su rentabilidad.

\section{Estudios relacionados}

\section{Descripción de las competencias gerenciales}

Elconcepto decompetencia para(HayGroup, 2017) está relacionada con "una característica personal que lleva a un comportamiento diferenciador de éxito", donde la empatía, las expectativas positivas y la identificación de redes de influencia, permite un mejor desempeño en las personas, este autor se basa en la teoría de la necesidades del psicólogo estadunidense David McClelland, donde define que el ser humano se interesa por: alcanzar logros, adquirir poder y el deseo de afiliación es decir, el relacionarse con los que lo rodean y que influyen en un clima organizacional, lleva a exigirse, a definir objetivos, para alcanzar un mejor desempeño.

De acuerdo con (HayGroup, 2017), complementando lo anterior, para el desempeño de las competencias se requiere adicionalmente de actitudes y aptitudes, dos elementos que se describen a lo largo del presente artículo con diferentes autores, en donde éstos son el factor común de las competencias, descritas en otros términos y características; de manera integral a la formación en competencias es necesario valores y rasgos motivacionales, un ingeniero debe visualizarse de manera holística y no como un operario que escucha órdenes y las ejecuta.

Otros autores describen las competencias de la siguiente manera, para (Corredor, Fontanilla, \& Escobar, 2015), existen las genéricas:

Para Castrillón \& Cabeza (2010, p. 19) "las competencias genéricas corresponden a las capacidades y habilidades compartidas o comunes a cualquier campo de estudio como la ética profesional, compromiso con la calidad, habilidades de interpretación, de análisis, de resolución de problemas, entre otras". Además, González et al, (2007), señala que las competencias se originan en diferentes "unidades del curso y son evaluadas en diferentes etapas. Pueden estar divididas en competencias relacionas con un área de 
conocimiento, competencias específicas para un campo de estudio y competencias genéricas que son comunes para diferentes campos de estudio" (Pág. 5)

Por otro lado (Asociación Colombiana de Fcultadoes de Administración-ASCOLFA, 2011) define competencias específicas y genéricas, las primeras relacionadas con un conocimiento disciplinar orientado a tareas para el desempeño en un área; y las segundas están enfocadas hacia las actitudes de la persona.

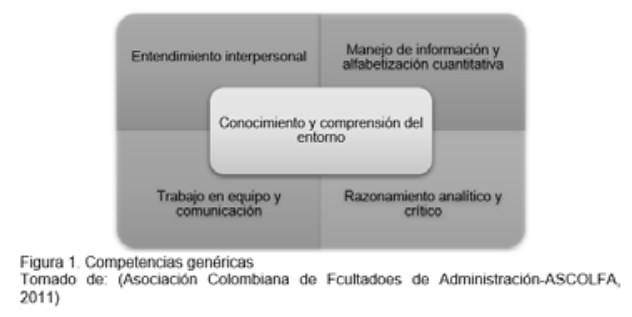

Las anteriores competencias permiten el desenvolvimiento en cualquier campo o área, que va desde entendimiento interpersonal, trabajo en equipo, pensamiento creativo para abordar problemáticas, empleando el liderazgo y la comunicación, entre otros, así como el manejo del segundo idioma y de las tecnologías de la información y comunicación-TIC.

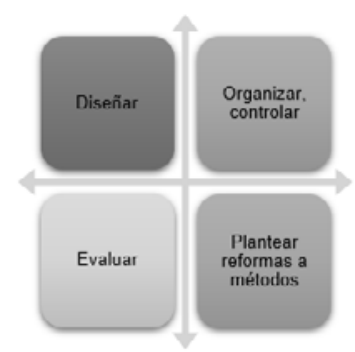

Figura 2. Algunas competencias genéricas Tomado de: (Asociación Colombiana de Fcultadoes de Administración-ASCOLFA 2011)

Las competencias específicas, se enfocan en la adquisición del conocimiento propio a la ingeniería a desempeñar; estas competencias deben desarrollar una secuencia de estapaspara los procesos, gestionar recursos y hacer seguimiento a proyectos, adicionalmente liderar grupos, tomar decisiones asertivas, para generar emprendimiento, innovación y pensamiento global, manejo de competencias digitales, para negociación por medio de la internet y así para dar alcance a resultados organizacionales y cumplimiento de la misión en las empresas.

\section{Modelo de competencias gerenciales}

La (Centeno \& Serafin, 2006) realizó un estudio sobre el modelo de competencias "estudiar los distintos modelos de competencia disponibles se concluye la ausencia un modelo único, superior e indiscutible con este fin", en la revisión documental encontraron que no existe un modelo único o estandarizado para ser aplicado a las organizaciones o en la gerencia de proyectos, las habilidades o competencias para elaborar proyectos, se ajustan a los perfiles del gerente líder y del equipo de trabajo, "cada contexto industrial posee sus propias especificidades que demandan esfuerzos, conocimientos y actitudes diferentes por parte del director del equipo"

Por lo anterior, el propósito del estudio de esta universidad, es proponer un modelo más concreto y más amplio para la gerencia de proyectos donde se adecue a los requerimientos empresariales tomando en cuenta su entorno, su personal y la calidad de los bienes o servicios que ofrecen, este modelo que proponen debe estar articulado con los procesos de aprendizaje, puesto que es importante conocer cómo los estudiantes de los programas de ingeniería aprenden, para diseñar estrategias que permitan el alcance de la adquisición de habilidades gerenciales.

Otro modelo para enseñar competencias gerenciales es el PMI (Project Management Institute), éste maneja tres dimensiones para las competencias, a) conocimiento de gerencia de proyectos y comprensión de la gerencia, b) desempeño en gerencia de proyectos y c) competencias personales, éstas dimensiones tienen agrupadas entre nueve y doce competencias para un gerente de proyectos y que a su vez tienen unos criterios de evaluación, para revisar o calificar el desempeño en cada una de ellas, reuniendo de 
manera clara el concepto de competencia desde la técnica, el conocimiento, manejo de herramientas, actitudes y comportamientos.

En la (Páez, 2005), se desarrolló una investigación referente a la identificación y reconocimiento de las competencias que se encuentran asociadas a la actividad gerencial en las organizaciones de los diferentes sectores económicos. Para esto, se hizo una caracterización sobre liderazgo, competencia, competencia laboral y elementos de las competencias desde el aspecto teórico, cuyo referente fue el Ministerio de Comercio, industria y turismo y otro desde el punto de vista académico, realizando el estudio a partir de la percepción de la Facultad de administración de empresas, del grupo de investigación Centro de liderazgo y de los estudiantes de pregrado del programa de administración de empresas de sexto semestre, se identificó que los estudiantes en sus espacios académicos adquieren los conocimientos técnicos y procedimentales, pero lo importante es saber hasta qué nivel alcanzan éstos conocimientos y qué tanto han sido asimilados, este proceso no se plantea en el aula.

\section{Experiencias académicas}

En un estudio académico realizado por (Ugueto \& Cardozo, 2010), se analizó la importancia del papel de la universidad frente a generar conocimiento y destrezas para el desarrollo de un país involucrando en un tema fundamental como es el desarrollo endógeno, estrategia de las organizaciones, para potenciar las capacidades internas de una nación, región o ciudad, para esto el currículo cumple un papel esencial para identificar competenciasindividuales, orientados a la contribución con el desarrollo endógeno, materia de interés de la investigación de los autores, para diseñar una propuesta metodológica que permita el diseño de una ruta de proyecto formativo, afianzando las competencias como un compromiso que se le garantiza al sector productivo y así responder a sus necesidades en cuanto a perfiles laborales gerenciales y administrativos.
Para esta propuesta académica de la investigación se desarrolló bajo el tipo de estudio de proyecto factible, definida por la Universidad Pedagógica Experimental que permite la elaboración de un modelo para abordar problemas de organizaciones o grupos sociales; con un enfoque cualitativo porque se realiza análisis por medio de revisiones documentalesydecampo,sobre perfiles requeridos para poder diseñar un currículo estructurado por competencias individuales gerenciales orientado hacia la capacidad de intervenir en el desarrollo endógeno.

Las instituciones de educación superiorestán en la tarea de definir metodologías que permitan un mejor aprendizaje en competencias gerenciales para los programas de ingenierías, para que les permita abordar otros campos de acción, como lo es gestionar proyectos de inversión, industriales, productivos, de innovación y de desarrollo tecnológicos.

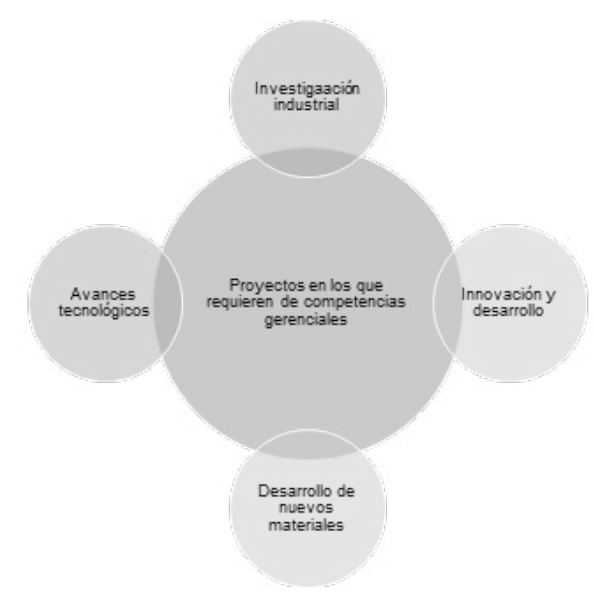

Figura 3. Proyectos para el campo ingenieril Tomado de: elaboración propia.

\section{Método}

Para el desarrollo del estudio, se trabajó bajo el enfoque cualitativo con un componente descriptivo (Lerma,2012),quepermitiórealizarunaexploración de documentos científicos y académicos donde describen las competenciasgerencialesyque fueronde guía para caracterizar las competencias pertinentes para ingenieros; desde lo descriptivo (Bernal, 2010), se definieron categorías de análisis, 
a partir de la revisión documental, para orientar las habilidades que los ingenieros requieren para formular y ejecutar proyectos empresariales.

Para analizar la información es necesario categorizar las variables del estudio, de manera que se unifiquen los criterios de descripción (Noguero, 2009) "Se denominan categorías a cada uno de los elementos o dimensiones de las variables investigadas y que van a servir para clasificar o agrupar según ellas las diversas unidades"

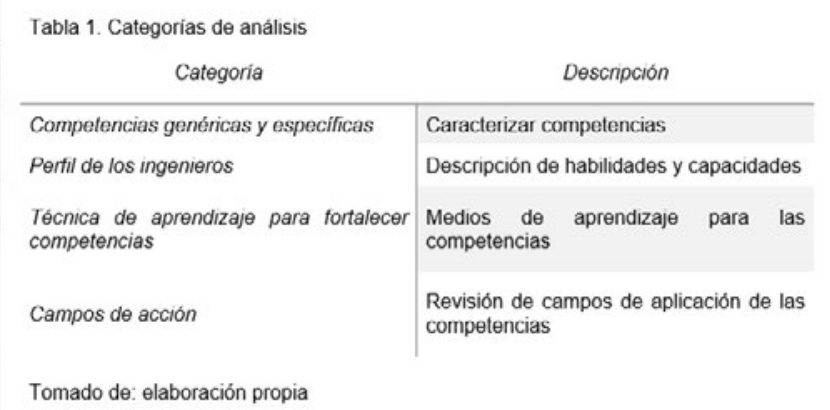

Tomado de: elaboración propia

A partir de las categorías, se puede presentar un análisis del estudio, con el fin de aportar al fortalecimiento de las competencias gerenciales de los ingenieros,potenciando el desarrollo de proyectos al interior de las empresas y de la academia, puesto que requieren del componente investigativo en ellos, para intervenir en factores como la innovación, avances en la ciencia y en el desarrollo tecnológico, más allá de sus funciones diarias, como supervisión de funciones, gestión productiva y liderazgo en los equipos de trabajo.

Para la orientación de esta investigación, se tomó como base documental el suministrado por Plan Nacional de Desarrollo 2018-2022 (Departamento Nacional de PLaneación, 2019), donde plantean retos y estrategias, en pro al desarrollo del país, aportando mejoras al sector empresarial, comercial y a la calidad de vida de los ciudadanos; para esta entidad es importante el planteamiento de objetivos y estrategias que permitan el crecimiento económico "aspiramos que Colombia alcance un ingreso per cápita a los 2.500 dólares anuales, que nos ubique en el grupo de países de ingresos altos".

Para esto, es necesario fortalecer el emprendimiento, la productividad, competitividad y el desarrollo de la ciencia y tecnología, lo cual se logra potenciando a ingenieros para que creen nuevos bienes o servicios o para generar más valor a los existentes, todo esto se alcanza a trabajar, cuando éstos profesionales potencializan sus habilidades para liderar proyectos, como los que se citaron en el presente artículo y que contribuyen al crecimiento de las empresas y por su puesto del país.

\section{Resultados}

La recolección de información se realizó de fuentes verídicas, que permitieron el análisis sobre conceptos referentes a competencias gerenciales y sobre la importancia de fortalecerlas en los profesionales del área de ingenierías, puesto que éstos perfiles sólo desarrollan proyectos en un $50 \%$ en el sector productivo y un $20 \%$ en el sector académico y que se requiere se incrementen éstos porcentajes para fomentar el desarrollo de la innovación y de las tecnologías, para mejorar procesos tanto productivos como organizacionales en las empresas. La economía Colombiana es impulsada en un 80\% por empresas PYMES (pequeñas, y medianas empresas), lo que significa que necesitan apoyo ingenieril para incrementar su productividad en recursos y procesos, para reducir la tasa de decadencia, que aunque fortalecen el país, tienen herramientas que requieren ser fortalecidas para mantenerse en los mercados comerciales y para expandirse a otros aún no explorados por parte de su competencia.

Se encontró que tanto empresas como instituciones educativas, las primeras requieren de ingenieros con capacidad de asumir retos y afrontar necesidades, empleando sus capacidades y habilidades disciplinares y también destrezas para el desenvolvimiento interpersonal, importantes 
para la gestión en las empresas y las segundas, su necesidad es formar ingenieros desde su pregrado y desde sus actualizaciones académicas en pos grados, en competencias gerenciales, para que administren y gestionen proyectos benéficos para las organizaciones y comunidades en general.

Laimportanciadetratareltemadecompetenciases para mostrarle diferentes visiones de desempeño a los ingenieros y que las competencias gerenciales son herramientas que permiten explorar, indagar, investigar y analizar entornos y situaciones en el que presentan problemáticas o necesidades, que requieren ser abordadas; un ingeniero debe estar en la capacidad de contribuir con el desarrollo de una comunidad que requiera de orientación y asesoría hacia la formulación y evaluación de proyectos sociales, que estén a favor de los beneficios de los ciudadanos y grupos vulnerables, que requieran de inclusión, a partir de propuestas empresariales, productivas e ingenieriles para su calidad de vida y la de los demás.

Un aporte importante de la academia es brindar capacitaciones periódicas a ingenieros una vez graduados, paraquemantengancomoherramienta importante el manejo de competencias, esto les permitirámejorar sus perfiles, contribuir a mejoras, diseñar nuevos esquemas y productos, ofrecer un valor agregado a empresas y comunidades y desde el aula trabajar con aprendizaje basados en problemas-ABP, para que los estudiantes contextualicen las competencias y a partir de éstos ejercicios manejarlas de acuerdo al caso a tratar.

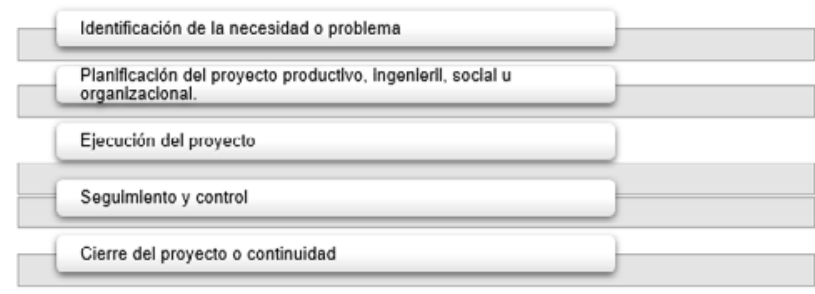

Figura 4 Fases de los proyectos como guía de aprendizaje para trabajar competencias gerenciales

Fuente: elaboración propia

\section{Conclusiones}

La investigación documental puedo determinar competencias pertinentes para los ingenieros, que se citan a continuación tanto genéricas como específicas, aproximadamente son treinta competencias, pero para una mejor comprensión sobre su pertinencia con éstos perfiles profesionales, se mencionarán algunos, para que sean empleados en el ejercicio laboral.

A continuación, se exponen algunas competencias que un ingeniero puede manejar para el desarrollo de proyectos, que son una guía para orientar el perfil como gerente en proyectos, esto le permitirá determinar, cuáles son las competencias necesarias de acuerdo con sus necesidades laborales:

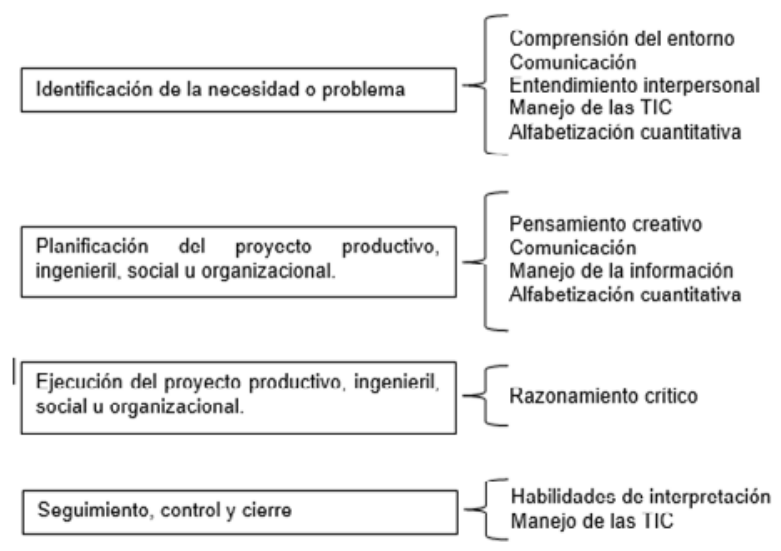

Figura 5. Competencias gerenciales específicas para cada fase de un proyecto

Fuente: elaboración propia a partir de (Murcia, Catumba, \& Velandia, 2018)

Figura 5. Competencias gerenciales específicas para cada fase de un proyecto Fuente: elaboración propia a partir de (Murcia, Catumba, \& Velandia, 2018)

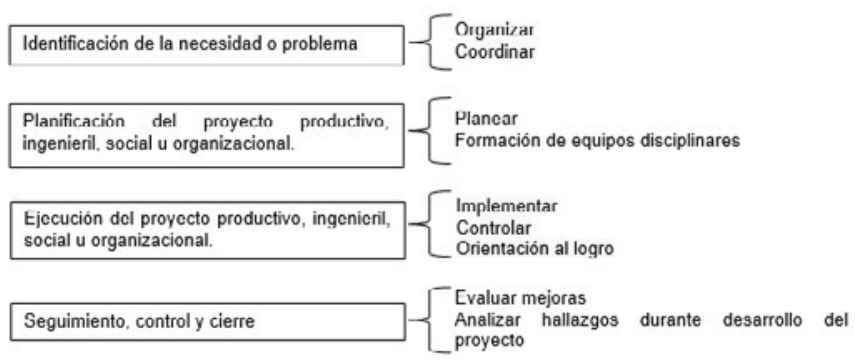




\section{Referencias bibliográficas}

Aguirre, C. A. (2016). Desarrollo de competencias de investigación en estudiantes de educación superior con la mediación de herramientas de m-Learning \& e-Learning (Development of research competencies in higher education students). Inclusión y Desarrollo. elSSN: 2590-7700, 69-83.

Asociación Colombiana de Fcultadoes de Administración-ASCOLFA. (2011). Características de los programa de administración en términos de competencias que permitan la evalaución en la pruebas Saber Pro. Bogotá: http://www.ascolfa.edu.co/web/archivos/saberpro/competencias_administracion_260711. pdf.

Bernal, C. (2010). Metodología de la investigación, administración, economía, humanidades y ciencias sociales. Bogotá: Pearson.

Caballero, S. L. (2001). Las competencias gerenciales en tiempos de virtualización. Revista Asuntos, 26. Castrillón, J., Cabeza, L., \& Lombana, J. (2015). Competencias más importantes para la disciplina administrativa en Colombia. Revista Contaduría y Administración. ISSN: 0186-1042, 777-795.

Centeno, R., \& Serafin, M. (2006). Modelo de Competencias para el Diseño de Programas de Formación de Gerentes de Proyectos. 4th Latin American and Caribbean Conference for Engineering and Technology (pág. 11). Caracas: Latin American and Caribbean Conference for Engineering and Technology-LACCEI. ISBN 0-9753970-2-X.

Contraloría General de la Respública. (2010). PLAN PLURIANUAL DE CAPACITACIÓN INTERNA. Costa Rica: http://www.oas.org/juridico/pdfs/mesicic4_cri_plan.pdf.

Corredor, A. D., Fontanilla, A. C., \& Escobar, J. V. (2015). COMPETENCIAS ESPECÍfICAS DE LOS ADMINISTRADORES COMO: FACTOR DE DESARROLLO. Brranquilla: https:/www.uac.edu.co/images/ stories/publicaciones/revistas_cientificas/dimension-empresarial/volumen-13-no-2/articulo15.pdf. Departamento Nacional de PLaneación. (2019). Bases del Plan Nacional de Desarrollo: pacto po Colombia, pacto por la equidad. Bogotá: Grupo de Comunicaciones .

HayGroup. (2017). Programas de formación para el desarrollo de competencias. Estados Unidos: https:// www.yumpu.com/es/document/read/22192105/para-el-desarrollo-de-competencias-hay-group.

Lerma, H. (2012). Meotodlogía de la invesitgación. Propuesta, anteproyecto y proyecto. . Bogotá: Ecoediciones. ISBN 978-958-648-602-6.

Murcia, J., Catumba, J., \& Velandia, D. (2018). SER GERENTEModelo de formación de líderes empresariales. Bogotá: Centro Editorial UNIMINUTO. ISBN: 978-958-763-264-4.

Noguero, F. L. (2009). El análisis de contenido como método de investigación. Revista de Educación. ISSN: 1575-0345, 167-179.

Páez, I. (2005). Competencias para el liderazgo gerencial. Primer Congreso Iberoamericano de Investigación en Administración (págs. 79-97). Manizales: niversidad Nacional de Manizales.

Roldán, I. G. (2012). Competencias profesionales: una propuesta de evaluación para las facultades de ciencias administrativas. Revista Educación y Educadores. ISSN: 0123-1294, 45-66.

The In stitute for Business and Finance Research. (2012). Global Conference on Business and Finance Proceedings. Global Conference on Business and Finance Proceedings. ISSN 1931-0285 (págs. 60-80). San Josè, Costa Rica: The In stitute for Bu siness and Finance Res earch.

Ugueto, M. G., \& Cardozo, N. (2010). Competencias individuales y el currículo universitario ante el reto del desarrollo endógeno. Visión gerencial. ISSN 1317-8822, 407-429.

Universidad Externado de Colombia. (2002). Competencias para el liderazgo gerencial. Bogotá: http:// revistas.uexternado.edu.co/index.php/sotavento/article/viewFile/1606/1445. 